
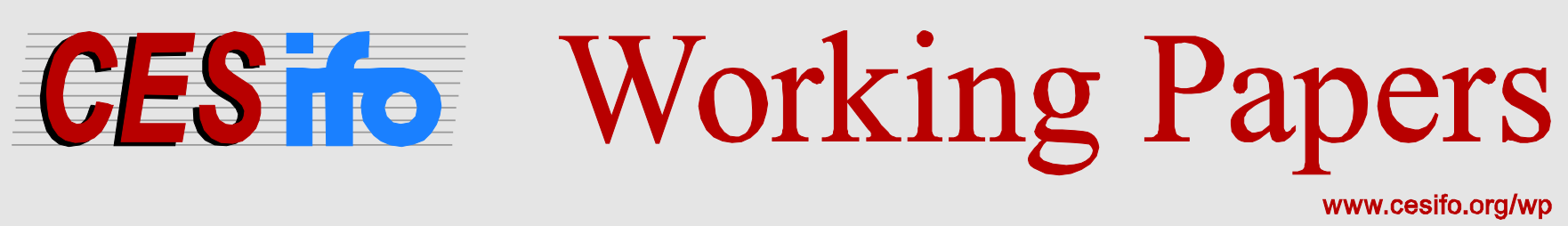

\title{
Technological Transfers in Global Climate Policy
}

\author{
Wolfgang Buchholz \\ Lisa Dippl \\ Michael Eichenseer
}

\author{
CESIFO WORKING PAPER NO. 5548 \\ CATEgORY 10: ENERGY AND ClimATE ECONOMICS \\ OCTOBER 2015
}
An electronic version of the paper may be downloaded
- from the SSRN website: Www.SSRN.com
- from the RePEc website: Www.RePEc.org
- from the CESifo website: www.CESifo-group.org/wp




\title{
Technological Transfers in Global Climate Policy
}

\begin{abstract}
Theoretical analysis and empirical evidence show that leadership behavior in climate policy through increased abatement efforts or international transfers cannot be expected to be very successful. In this paper we instead show that pioneering activities, which are based on green technological innovations carried out by a coalition of countries, may be a better approach for combatting global warming through unilateral action. In particular, we examine in an otherwise standard model of private public good supply how the success of such a policy depends on the intensity and scope of technological spillovers.
\end{abstract}

JEL-Code: H410, H870, O310, Q540, Q550.

Keywords: climate policy, green technological innovations, voluntary public good provision, leadership.

\author{
Wolfgang Buchholz* \\ Department of Economics \\ University of Regensburg \\ Germany - 93040 Regensburg \\ wolfgang.buchholz@ur.de
}

Lisa Dippl

Faculty of Business, Economics and

Management Information Systems

University of Regensburg

Germany - 93040 Regensburg

lisa.dippl@wiwi.uni-regensburg.de
Michael Eichenseer

Department of Economics

University of Regensburg

Germany - 93040 Regensburg

Michael.Eichenseer@wiwi.uni-

regensburg.de

*corresponding author

This Version: September 30, 2015

We gratefully acknowledge financial support by the German Federal Ministry of Research through the research project ECCUITY (FKZ 01LA1104B). 


\section{Introduction}

Progress in global climate policy is impeded by the enormous difficulties to ensure international cooperation and coordination on greenhouse gas mitigation, i.e. to conclude an international climate agreement which requires ambitious and effectively binding obligations and which is stable (see, e.g., Sandler, 2004, and Finus, 2001, for a detailed theoretical analysis of these problems). The hope therefore is that - following a "bottom up approach" - pioneering activities of a coalition of leading countries might help moderate global warming and thus increase the supply of this presumably most important public good. When such leading activities are considered, the focus normally is on increased abatement efforts or transfers to outsider countries, which in a standard model of public good provision have equivalent effects (see e.g. Cornes and Sandler, 2000, for an analysis of this equivalence). But such unilateral measures are only of limited effectiveness as the countries outside the coalition can be expected to show some "crowdingout" behaviour, i.e. to decrease their public good contributions as a reaction to the increased contributions made by the coalition. In Sinn's (2012) figurative terminology these outsider countries thus are "grabbing from the collection box" which reduces the positive effect on public good supply resulting from the pioneering activities (see e.g. Buchholz, Haslbeck and Sandler, 1998). Even worse, it is even possible that total public good supply falls when countries unilaterally raise their abatement efforts before they enter climate negotiations (see Hoel, 1991).

In the face of these obstacles for successful leadership in public good provision (for an overview see Buchholz and Sandler, 2015) this paper considers another type of pioneering activities which is not based on an increase of public good contributions. Rather, leading behaviour manifests itself in investments to improve "green" technologies (such as renewable energies or energy efficiency measures) which help reduce abatement costs and thus make the production of the global public good less costly. It is well-known (see Buchholz and Konrad, 1994, and Ihori, 1996) that in the context of voluntary public good provision it may harm a country if it unilaterally reduces its cost for public good provision even in the extreme case where the innovation is completely costless. Then environmentally friendly technological progress is blocked on the basis of strategic reasons. In this paper we extend this analysis on the strategic choice of abatement technology which leads us to a more optimistic picture: If a country or a coalition of countries is - as some kind of substitute for monetary transfers - able to make the fruits of its inno- 
vation available to other countries free of charge this will not only be beneficial for these countries but also for the pioneering coalition itself. This in turn makes the coalition more prepared to engage in green R\&D-activities.

The analysis will be carried out in the standard framework of voluntary public good provision (as exposed by Bergstrom, Blume and Varian, 1986, and Cornes and Sandler, 1996). But unlike these traditional contributions to the theory of public goods we will make use of the more recent Aggregative Game Approach (see Cornes and Hartley, 2007). Application of this approach considerably facilitates the analysis of Nash equilibria in games of public good provision which are quite complex in the scenario considered in this paper.

\section{The Framework}

Let there be $n$ countries $i=1, \ldots, n$ be given which all have the same initial endowment $w$ and the same utility function $u\left(x_{i}, G\right)$ where $x_{i}$ denotes private consumption of country $i$ and $G$ is public good supply. Especially in the context of climate change $G$ can be interpreted as the amount of greenhouse gases totally avoided. The utility function is assumed to have the standard properties, i.e. it is twice partially differentiable with the first partial derivatives $u_{1}\left(x_{i}, G\right)>0$ and $u_{2}\left(x_{i}, G\right)>0$ and quasi-concave. Moreover, we suppose that the private and the public good both are strictly non-inferior.

The crucial element for our analysis is that the countries may differ in their productivities for generating the public good which are represented by the country-specific marginal rates of transformation $m r t_{i}$ between the private and the public good $a_{i}$ : This productivities indicate how many units of the public good country $i$ can produce if it spends one unit of the private good for public good provision. The reciprocal value $c_{i}$

$=\frac{1}{a_{i}}$ then indicates how many units of the public good country $i$ has to give up in order to get one additional unit of the public good. In the case of environmental public goods $c_{i}$ thus represents the marginal abatement costs of country $i$.

Under these assumptions a feasible allocation $\left(x_{1}, \ldots, x_{n}, G\right)$ has to satisfy the aggregate budget constraint

$$
G=\sum_{i=1}^{n} a_{i}\left(w-x_{i}\right)
$$


or, equivalently,

$$
G+\sum_{i=1}^{n} a_{i} x_{i}=\sum_{i=1}^{n} a_{i} w
$$

In order to describe the Nash equilibria of voluntary public good provision in this setting by means of the Aggregative Game Approach let $e\left(G, \alpha_{i}\right)$ be country $i$ 's (income) expansion where $\alpha_{i}$ denotes the marginal rate of substitution $m r s_{i}$ between the private and the public good. As non-inferiority of both goods is assumed these expansion paths are well-defined and strictly monotone increasing in $G$. Along an expansion path $e\left(G, \alpha_{i}\right)$ the indifference curves of a country all have the same slope $-\alpha_{i}$. As an additional assumption regarding preferences we assume that $e\left(0, \alpha_{i}\right)=0$ and $\lim _{G \rightarrow \infty} e\left(G, \alpha_{i}\right)=\infty$.

The essential point for the characterization of Nash equilibria is that a country which makes a strictly positive public good contribution is in an individual equilibrium position only if its $m r s_{i}$ coincides with its $m r t_{i}$, i.e. $\alpha_{i}=a_{i}$ holds. Otherwise, country $i$ could attain a higher utility level either by slightly increasing (if $\alpha_{i}>a_{i}$ ) or by slightly decreasing (if $\alpha_{i}<a_{i}$ ) its public good contribution. This, however, means that in an interior Nash equilibrium $\left(\hat{x}_{1}, \ldots, \hat{x}_{n}, \hat{G}\right)$ with positive public good contributions of all countries, country $i$ 's position $\left(\hat{x}_{i}, \hat{G}\right)$ has to lie on the expansion path $e\left(G, a_{i}\right)$ such that $\hat{x}_{i}=e\left(\hat{G}, a_{i}\right)$.

As a benchmark we consider the case in which the productivity parameters $a_{i}$ are fixed and no country undertakes efforts to improve public good productivity. Then no $\mathrm{R} \& \mathrm{D}$-costs have to be taken into account, and the budget constraint (1) for an interior Nash equilibrium becomes

$$
\hat{G}=\sum_{i=1}^{n} a_{i}\left(w-e\left(\hat{G}, a_{i}\right)\right)
$$

Given our assumptions the function $\Phi(G):=\sum_{i=1}^{n} a_{i}\left(w-e\left(G, a_{i}\right)\right)$, whose value at $\hat{G}$ appears on the right hand side of eq. (3), is strictly monotone increasing and continuous and, given our assumptions on expansion paths, has $\Phi(0)=0$ and $\lim _{G \rightarrow \infty} \Phi(G)=\infty$. Hence, 
by the intermediate value theorem there exists exactly one level of public good supply $\hat{G}$, which fulfils condition (3). If $e\left(\hat{G}, a_{i}\right)<w_{i}$ holds for each country $i=1, \ldots, n$, the Nash equilibrium is interior with public good supply $\hat{G}$ and private consumption levels $\hat{x}_{i}=e\left(\hat{G}, a_{i}\right)$. In this Nash equilibrium country $i=1, \ldots, n$ spends $\hat{z}_{i}=w_{i}-\hat{x}_{i}$ on the public good thus inducing an increase of public good supply by $\hat{g}_{i}=a_{i} \hat{z}_{i}$.

\section{Technological Interdependencies}

We start from a situation in which all countries have the same productivity parameter $a_{0}$. Public good supply $\hat{G}\left(a_{0}\right)$ in the Nash equilibrium - which is clearly interior in this initial state of full symmetry and without any R\&D-costs - then is given by

$$
\hat{G}\left(a_{0}\right)=n a_{0}\left(w-e\left(\hat{G}\left(a_{0}\right), a_{0}\right)\right)
$$

Now the possibility arises that a subgroup of countries undertakes some R\&D-efforts aimed at improving "green" technologies (such as better insulation of houses, renewable energies, smart grids and new methods for power storage) through which the reduction of carbon emissions becomes cheaper or, in other words, the productivity of the global public good climate protection is increased. Through intended or unintended technological spillovers other countries may also benefit from these productivity enhancing effects even if they do not incur any of the costs associated with developing these ecologically friendly technologies.

For a precise description of this scenario we divide the whole group of $n$ countries into three subgroups $\mathrm{K}, \mathrm{L}$ and $\mathrm{M}$ whose members are playing a two-stage game.

\section{Subgroup K consisting of $k$ countries}

The members of subgroup $k$ are forming a technological coalition which is willing to play a pioneering role in climate policy by collectively promoting green innovations. In the framework of our model this means that at the first stage of the game coalition $\mathrm{K}$ is able to choose an improved production technology for the public good which exhibits a higher public good productivity $a$ than the original technology. Choosing some $a>a_{0}$, however, is not costless but results in R\&D-costs of $c_{k}(a)$ for each country in coalition K. This cost function is assumed to be differentiable in $a$ and has $c_{k}\left(a_{0}\right)=0$. If, as in the 
case of basic research, R\&D-costs can be divided among the members of the coalition $c_{k}(a)$ will - for any $a>0$ - fall when $k$ increases. However, if technological progress is based on learning-by-doing activities, which have to be carried out in each country of the coalition at an equal scale, then $c_{k}(a)$ will not be affected by the size of the coalition.

While the coalition cooperates at the innovation stage, the coalition members still act independently in the second stage of the game at which the coalition members decide on their contributions to the public good. This assumption reflects the notion that in climate policy cooperation on abatement levels is harder to achieve than technological cooperation.

\section{Subgroup L consisting of $l$ countries}

The members of subgroup L do not have a share in the spillover: They stick to the original technology with the productivity parameter $a_{0}$ irrespective of the technological choice made by coalition K. This inability to make use of the better environmental technologies may arise from specific physical or meteorological conditions. E.g. countries in the tropical zones obviously do not benefit from improved efficiency in the heating of buildings, and countries like Canada with fewer sunshine hours than Florida cannot gain much from the development of solar technology. But it is also possible that in developing countries the capacities for adopting the improved technologies are lacking. In contrast to the physical limitations these obstacles can be removed, e.g. through education and the formation of human capital.

\section{Subgroup M consisting of $m$ countries}

For subgroup $M$ there is a technological spillover from the technological innovations provided by coalition $\mathrm{K}$ so that they become more productive in generating the public good - but possibly to a different degree as the coalition members. The differentiable function $b(a)$ describes which productivity parameter results in each country in M when the productivity parameter chosen in coalition $\mathrm{K}$ is $a$. This function measuring the intensity of the spillover effect is monotone increasing in $a$ with $b\left(a_{0}\right)=a_{0}$. The normal case will be $b^{\prime}(a) \leq 1$, which means that the countries in M benefit not more from the innovation than the countries in K. Nevertheless, situations are conceivable in which $b^{\prime}(a)>1$ holds so that the productivity increase for subgroup $\mathrm{M}$ is even larger than in $\mathrm{K}$. 
An example for this might be solar energy when in the countries of subgroup M solar radiation is stronger than in the countries of subgroup $\mathrm{K}$.

Like the countries in coalition $\mathrm{K}$ also the countries in the outsider subgroups $\mathrm{L}$ and $\mathrm{M}$ determine their public good contributions non-cooperatively at the second stage of the game.

Applying the Aggregative Game Approach, it now is straightforward to describe the interior Nash equilibrium which results when coalition $\mathrm{K}$ has chosen some productivity parameter $a \geq a_{0}$ as we know that in the Nash equilibrium

- the position of all countries in $\mathrm{K}$ is on the expansion path $e(G, a)$.

- the position of all countries in $\mathrm{L}$ is on the expansion path $e\left(G, a_{0}\right)$.

- the position of all countries in M is on the expansion path $e(G, b(a))$.

Based on condition (3) public good supply $\hat{G}(a)$ in the Nash equilibrium if coalition K has chosen the productivity parameter $a$ is characterized by the following equation:

$$
\hat{G}(a)=k a\left(w-e(\hat{G}(a), a)-c_{k}(a)\right)+l a_{0}\left(w-e\left(\hat{G}(a), a_{0}\right)\right)+m b(a)(w-e(\hat{G}(a), b(a))) .
$$

Private consumption of the countries in subgroups $\mathrm{K}, \mathrm{L}$ and M thus is $\hat{x}_{K}(a)=e(\hat{G}(a), a)$, $\hat{x}_{L}(a)=e\left(\hat{G}(a), a_{0}\right)$ and $\hat{x}_{M}(a)=e(\hat{G}(a), b(a))$, respectively. Note that in eq. (5) it is taken into consideration that the members of group $\mathrm{K}$ do not spend the whole residual between income and private consumption for public good provision because they have to spend $c_{k}(a)>0$ for R\&D-efforts when choosing some $a>a_{0}$.

Since the initial Nash equilibrium is interior it follows from a standard continuity argument that the Nash equilibrium will stay interior when the productivity parameter $a$ chosen by coalition $\mathrm{K}$ is sufficiently close to $a_{0}$. The analysis to follow only considers these cases.

\section{The Change in Public Good Supply through Technological Progress}

Let the partial derivative of any expansion path $e(G, \alpha)$ w.r.t. public good supply $G$ be denoted by $e_{1}(G, \alpha)$ which describes how private consumption changes if one is moving 
along an expansion path. Analogously, $e_{2}(G, \alpha)$ is the partial derivative of the expansion path w.r.t. to the marginal rate of substitution $\alpha$. This derivative indicates the change of private consumption which results when - for a given level of public good supply - the move is to another expansion path corresponding to a higher marginal rate of substitution. From the non-inferiority assumption on preferences we have $e_{1}(G, \alpha)>0$ and $e_{2}(G, \alpha)<0$.

To calculate the effect on public good supply $\hat{G}^{\prime}(a)=\frac{\partial \hat{G}}{\partial a}$ which is driven by a marginal change of its productivity parameter by coalition $\mathrm{K}$ we first consider the total differential of eq. (5) at some arbitrary $a$ for which interiority holds which yields

$$
\begin{aligned}
\hat{G}^{\prime}(a)= & -k\left(w-e(\hat{G}(a), a)-c_{k}(a)\right)-k a\left(e_{1}(\hat{G}(a), a) \hat{G}^{\prime}(a)+e_{2}(\hat{G}(a), a)-c_{k}^{\prime}(a)\right) \\
& -\operatorname{lae}_{1}(\hat{G}(a), a) \hat{G}^{\prime}(a) \\
& +m b^{\prime}(a)(w-e(\hat{G}(a), b(a)))-m b(a)\left(e_{1}(\hat{G}(a), b(a)) \hat{G}^{\prime}(a)+e_{2}(\hat{G}(a), b(a)) b^{\prime}(a)\right) .
\end{aligned}
$$

We now apply eq. (6) to infer the effects on public good supply which result from a marginal change of $a$ starting from $a_{0}=b\left(a_{0}\right)$. Without loss of generality we can assume $a_{0}=1$ and, to simplify notation, we use abbreviations as follows: $\hat{G}^{\prime}=\hat{G}^{\prime}(1)$, $\hat{z}=w-e(\hat{G}(1), 1), \kappa_{k}=c_{k}^{\prime}(1), \beta=b^{\prime}(1), \gamma_{1}=e_{1}(\hat{G}(1), 1)$ and $\gamma_{2}=e_{2}(\hat{G}(1), 1)$. Since $c_{k}(1)=0$ by assumption condition (6) then turns into

$$
\hat{G}^{\prime}=k\left(\hat{z}-\gamma_{1} \hat{G}^{\prime}-\gamma_{2}-\kappa_{k}\right)-l \gamma_{1} \hat{G}^{\prime}+m\left(\beta \hat{z}-\gamma_{1} \hat{G}^{\prime}-\gamma_{2} \beta\right) .
$$

Solving (7) for $\hat{G}^{\prime}$ and observing $k+l+m=n$ gives the following result.

Proposition 1: If coalition K marginally increases its productivity parameter $a$ starting from the symmetric Nash equilibrium with $a_{0}=1$ then public good supply changes by

$$
\hat{G}^{\prime}=\frac{(k+m \beta)\left(\hat{z}-\gamma_{2}\right)-k \kappa_{k}}{1+n \gamma_{1}}
$$


Public good supply hence increases if and only if

$$
k \kappa_{k}<(k+m \beta)\left(\hat{z}-\gamma_{2}\right)
$$

Since $\gamma_{1}>0$ and $\gamma_{2}<0$ condition (9) directly shows that - for a given partition into the three subgroups - an increase in public good supply results if the aggregate marginal costs for the technological improvement $k \kappa_{k}$ are not too high. A high spillover parameter $\beta$ and a high public good contribution $\hat{z}$ in the original Nash equilibrium are also favourable for an increase of public good supply as both help to make the increase of public good productivity more effective.

If, however, the R\&D-costs are sufficiently high, so that $k \kappa_{k}>(k+m \beta)\left(\hat{z}-\gamma_{2}\right)$ holds, public good supply is reduced by the innovation. The reason for this adverse effect is that due to the costly R\&D-efforts coalition K's resources available for public good provision are reduced while at the same time the spillover effect is too weak, either because only few countries are positively affected or the intensity of the spillover is small.

In addition we can infer from conditions (8) and (9) how for a fixed total number of countries $n$ the size of the different subgroups affects the change of public good supply. In this context we first note that $\hat{z}, \gamma_{1}$ and $\gamma_{2}$ refer to the original fully symmetric Nash equilibrium and thus do not depend on $k, l$ and $m$ as long as the total number of countries $n=k+l+m$ is fixed.

Proposition 2: Assume that $\hat{G}^{\prime}$ is positive. Then $\hat{G}^{\prime}$ is the larger

- the larger the coalition $\mathrm{K}$ is when aggregate marginal costs $k \kappa_{k}$ of the technological improvement are not rising in $k$.

- the larger the group $\mathrm{M}$ is.

- the smaller $\gamma_{1}$ and the larger $-\gamma_{2}$ are.

In a Nash equilibrium public good supply normally is too low as compared to Pareto optimal levels (see Buchholz and Peters, 2001, for a treatment especially of exceptions). Against this background Proposition 2 says that this "underprovision" is mitigated both through a spatial expansion of the technological spillover, i.e. an increase of $m$, and an 
increase of its intensity $\beta$. The same positive effect on public good supply occurs if the coalition $\mathrm{K}$ is enlarged given that $\beta \leq 1$ and $k \kappa_{k}$ is decreasing in $k$.

For a further interpretation of Proposition 2 note that a small $\gamma_{1}$ means that in a $x_{i}$ $G$-diagram the income expansion path $e(G, 1)$ is relatively steep. Then along this expansion path an increase of public good supply is accompanied by a small increase in private consumption which is favourable for an increase of public good supply when $a$ is increased. The same holds true for a large value of $-\gamma_{2}$ which represents a strong shift of the expansion path to the left and thus a large increase of the willingness to pay for the public good.

As a next step we examine the incentives the coalition $\mathrm{K}$ has for making a green innovation through which its public good productivity is increased.

\section{The Incentives for Coalition $K$ to Make the Technological Improvement}

Given some productivity parameter $a$ utility of a member of coalition $\mathrm{K}$ is $\hat{u}_{K}=$ $u(e(\hat{G}(a), a), \hat{G}(a))$ in the Nash equilibrium as $e(\hat{G}(a), a)=\hat{x}_{K}(a)$ is its private consumption. A marginal variation of $a$ changes this utility by

$$
\hat{u}_{K}^{\prime}(a)=u_{1}\left(\hat{x}_{K}(a), \hat{G}(a)\right)\left(e_{1}(\hat{G}(a), a) \hat{G}^{\prime}(a)+e_{2}(\hat{G}(a))\right)+u_{2}\left(\hat{x}_{K}(a), \hat{G}(a)\right) \hat{G}^{\prime}(a) .
$$

Without loss of generality we can assume that at the original Nash equilibrium for $a_{0}=1$ we have $\hat{u}_{1}\left(\hat{x}_{K}(1), \hat{G}(1)\right)=\hat{u}_{2}\left(\hat{x}_{K}(1), \hat{G}(1)\right)=1$. With the abbreviations as introduced before and additionally letting $\hat{u}_{K}^{\prime}=\hat{u}_{K}^{\prime}(1)$ eq. (10) then is reduced to

$$
\hat{u}_{K}^{\prime}=\left(1+\gamma_{1}\right) \hat{G}^{\prime}+\gamma_{2}
$$

Based on eq. (11) a precise condition for an increase of utility for countries in the coalition $\mathrm{K}$ is provided by the next result. In its first part this Proposition is a direct consequence of eq. (11) and in its second part it follows from plugging $\hat{G}^{\prime}$ as given by eq. (8) into eq. (11). 
Proposition 3: Starting from the Nash equilibrium with $a_{0}=1$ the members of coalition $\mathrm{K}$ benefit from an increase of their public good productivity if and only if

$$
\hat{G}^{\prime}>\frac{-\gamma_{2}}{1+\gamma_{1}}>0
$$

holds or, equivalently, if and only if

$$
k \kappa_{k}<(k+m \beta)\left(\hat{z}-\gamma_{2}\right)+\gamma_{2} \frac{1+n \gamma_{1}}{1+\gamma_{1}}
$$

As $\gamma_{1}>0$ and $\gamma_{2}<0$ it follows from condition (12) that a higher public good supply is a necessary but not a sufficient condition for an increase of a coalition member's utility: The coalition members only benefit from their R\&D-efforts when the increase in public good supply is strong enough.

The factors which determine the right hand side of inequality (13) are similar to those characterizing the change of public good supply: An enlargement both of the coalition $\mathrm{K}$ and of the group $\mathrm{M}$ are favourable for an increase of utility for the members of $\mathrm{K}$. Concerning the incentives for innovation in $\mathrm{K}$ this in particular shows how important it is to ensure a broad dissemination of the improved technologies. Giving patents for green technological innovations to other countries away free of charge thus may be a clever strategic move for coalition $\mathrm{K}$.

Concerning the second term we note that $\frac{1+n \gamma_{1}}{1+\gamma_{1}}$ is increasing in $\gamma_{1}$. Hence, a utility increase for countries in coalition $\mathrm{K}$ is more likely if $\gamma_{1}$ is small. The effect of $\gamma_{2}$, however, is ambiguous.

\section{Utility Effects for the Outsiders}

We now examine how utility of the countries in the groups $\mathrm{L}$ and $\mathrm{M}$ is changed by the innovative activities of coalition $\mathrm{K}$.

Differentiating utility $\hat{u}_{L}(a)=u\left(\hat{x}_{L}(a), \hat{G}(a)\right)=u\left(e\left(\hat{G}(a), a_{0}\right), \hat{G}(a)\right)$ of a country in L and utility $\hat{u}_{M}(a)=u\left(\hat{x}_{M}(a), \hat{G}(a)\right)=u(e(\hat{G}(a), b(a)), \hat{G}(a))$ of a country in group M w.r.t. the productivity parameter $a$ yields 


$$
u_{L}^{\prime}(a)=u_{1}\left(\hat{x}_{L}(a), \hat{G}(a)\right) e_{1}\left(\hat{G}(a), a_{0}\right) \hat{G}^{\prime}(a)+u_{2}\left(\hat{x}_{L}(a), \hat{G}(a)\right) \hat{G}^{\prime}(a) .
$$

(15) $u_{M}^{\prime}(a)=u_{1}\left(\hat{x}_{M}(a), \hat{G}(a)\right)\left(e_{1}(\hat{G}(a), b(a)) \hat{G}^{\prime}(a)+e_{2}(\hat{G}(a), b(a)) b^{\prime}(a)\right)$

$$
+u_{2}\left(\hat{x}_{M}(a), \hat{G}(a)\right) \hat{G}^{\prime}(a)
$$

Assuming again $a_{0}=1$ and $u_{1}(\hat{x}(1), \hat{G}(1))=u_{2}(\hat{x}(1), \hat{G}(1))=1$ and abbreviating $\hat{u}_{L}^{\prime}=\hat{u}_{L}^{\prime}(1)$ and $\hat{u}_{M}^{\prime}=\hat{u}_{M}^{\prime}(1)$ a marginal change of productivity at the initial Nash equilibrium thus results in utility changes as follows:

$$
\begin{aligned}
& \hat{u}_{L}^{\prime}=\left(1+\gamma_{1}\right) \hat{G}^{\prime} \\
& \hat{u}_{M}^{\prime}=\left(1+\gamma_{1}\right) \hat{G}^{\prime}+\beta \gamma_{2} .
\end{aligned}
$$

Comparing the utility changes for the three groups $\mathrm{K}, \mathrm{L}$ and $\mathrm{M}$ as described by eqs. (11), (16) and (17) leads to the following result:

Proposition 4: If coalition $\mathrm{K}$ marginally increases its public good productivity starting from the Nash equilibrium with $a_{0}=1$ the countries in $\mathrm{K}$ benefit least while countries in the group L benefit most, i.e. $\hat{u}_{K}^{\prime} \leq \hat{u}_{M}^{\prime}<\hat{u}_{L}^{\prime}$.

The interpretation of Proposition 4 is as follows: Through the change of public good productivity in coalition $\mathrm{K}$ utility of countries in each subgroup is equally affected by $\left(1+\gamma_{1}\right) \hat{G}^{\prime}$, which is positive if public good supply increases. For countries in $\mathrm{K}$ there is, however, a negative partial effect on utility which is expressed by $\gamma_{2}<0$ and which reflects the increased willingness to pay for the public good when productivity improves. The same effect hits the group $M$ but to a lesser degree if the spillover is incomplete, i.e. $\beta<1$. If, however, $\beta=1$ the utility change is the same for group $\mathrm{K}$ and group $\mathrm{M}$ even though only the members of the coalition $\mathrm{K}$ initially bear the cost of the green innovation. This means that, due to equilibrium repercussions, R\&D-costs can be shifted to other countries. This indirect redistribution effect is, in a certain sense, similar to the famous Warr neutrality in voluntary public good provision (see Warr, 1982, and e.g. 
Cornes and Sandler, 1996) which in particular implies that in an interior Nash equilibrium an increase of income in some country will increase utility not only in that specific country but in all countries.

The negative effect, which arises from the change of the willingness to pay for the public good implied by the technological improvement, is completely absent for countries in group L whose technology is unaffected by the innovation. Therefore, the members of this group benefit most. This, however, creates an incentive problem because countries in group $\mathrm{K}$ attain a higher utility level if they do not adopt the better technology for public good provision. This strategic effect, however, is avoided if the technological spillover occurs automatically which, e.g., is the case if firms in coalition $\mathrm{K}$ are the dominant producers of energy technology and thus can set environmentally friendly standards worldwide (see, e.g., Barrett, 2003). For the countries in group M also cobenefits from climate friendly may arise which, on the one hand, may be caused by improved possibilities to abate locally damaging pollutants as, e.g. particulate matter from power plants (see, e.g., Finus and Rübbelke, 2013) and, on the other hand, by the prospect of initiating a sustainable growth process implied by the transition to a low-carbon economy (see Stern, 2015). In this way the adoption of green technologies is promoted. In the sense of "issue linkage" the coalition may also introduce separate incentive mechanisms (as, e.g., additional financial aid) to ensure broad dissemination of its green innovation.

Moreover, the countries outside the coalition $\mathrm{K}$ may notice that their unwillingness to apply the new technology can undermine the willingness of coalition $\mathrm{K}$ to make the R\&D-efforts. To prevent this undesirable outcome the outsiders also may form a separate coalition in which they commit themselves to adopt the improved technology.

\section{An Example}

We now specifically assume that $w=1$ and that all countries have the Cobb-Douglas utility function $u\left(x_{i}, G\right)=x_{i}^{\rho} G$. For some marginal rate of substitution $\alpha$ the expansion path is given by $e(G, \alpha)=\frac{\rho}{\alpha} G$ which gives $e_{1}(G, \alpha)=\frac{\rho}{\alpha}$ and $e_{2}(G, \alpha)=-\frac{\rho}{\alpha^{2}} G$. According to eq. (4) the symmetric Nash equilibrium at $a_{0}=1$ is given by the public good supply level 
$\hat{G}(1)=\frac{n}{n \rho+1}$, the private good consumption levels $\hat{x}(1)=\frac{n \rho}{n \rho+1}$ and country-specific public good contributions $\hat{z}(1)=\frac{1}{n \rho+1}$. Since $\gamma_{1}=\rho$ and $\gamma_{2}=-\frac{n \rho}{n \rho+1}$ we get

$$
\begin{gathered}
\hat{G}^{\prime}=\frac{k+m \beta-k \kappa_{k}}{n \rho+1} \\
\hat{u}_{K}^{\prime}=\frac{(\rho+1)\left(k+m \beta-k \kappa_{k}\right)-n \rho}{n \rho+1} \\
\hat{u}_{L}^{\prime}=\frac{(\rho+1)\left(k+m \beta-k \kappa_{k}\right)}{n \rho+1} \\
\hat{u}_{M}^{\prime}=\frac{(\rho+1)\left(k+m \beta-k \kappa_{k}\right)-n \rho \beta}{n \rho+1}
\end{gathered}
$$

We now especially look at eq. (19) and consider the extreme case when there is only a single pioneering country, i.e. $k=1$. The innovation is profitable for this country if its R\&D-costs are below a certain threshold level, i.e.

$$
\kappa_{1}<1+m \beta-\frac{n \rho}{\rho+1} .
$$

Now let either $m=0$ or $\beta=0$ so that there are no technological spillovers. Then, even if the innovation is completely costless, the potentially pioneering country has no incentive to increase its public good productivity if $1-\frac{n \rho}{\rho+1}<0$ or, equivalently, $n>\frac{\rho+1}{\rho}$, which is always the case if the total number of countries is sufficiently large. A single country then would even have an incentive to choose a technology with higher abatement costs, which is the paradoxical effect described by Buchholz and Konrad (1994).

But if in contrast there is a technological spillover the innovation will be profitable for the country if the technological spillover extends to sufficiently many countries, i.e. if 


$$
m>\frac{(n-1) \rho-1}{(\rho+1) \beta}
$$

Some values for $m$ which satisfy condition (23) exist if the right hand side of this inequality is smaller than $n-1$, i.e. if the spillover is sufficiently strong so that

$$
\beta>\underline{\beta}:=\frac{1}{n-1}\left(\frac{n \rho}{\rho+1}-1\right)
$$

holds. In the case of a perfect spillover condition (24) is always fulfilled. The example thus clearly illustrates how a single country's incentive to innovate depends on the number of followers and the strength of the spillover effect. For a further specification consider the case where $n=10$ and $\rho=1$. Then without a spillover a costless marginal increase of public good productivity does not pay for a single country. But if there is a spillover with $\beta=1$, which benefits at least five other countries, condition (24) implies that the innovation becomes worthwhile for the country which undertakes it. Moreover, the lower threshold for the productivity parameter which is obtained from condition (24) is $\underline{\beta}=\frac{4}{9}$.

\section{Conclusion}

In this paper we have shown how the provision of a global public good such as climate protection may be improved through unilateral action of a group of countries which collectively carry out a green technological innovation lowering the costs of providing the global public good, i.e. in the case of climate change the costs of greenhouse gas abatement. The success of such a specific form of leading behaviour not only is more likely if the cooperating coalition is large but also if there is a steep rise of public good productivity in as many other countries as possible, i.e. if the technological spillover effect is strong both at the intensive and at the extensive margin. A basic message of this paper is that it is not only favourable for the climate but also for the coalition if these follower countries get free access to the improved technology and thus receive some indirect transfer from the coalition. However, these recipient countries benefit less from the innovation than the complete outsiders that stick to the old high-cost technology. This 
creates an incentive problem for technology adoption so that it may become necessary to complement the unilateral R\&D-policy by additional mechanisms to ensure a farreaching diffusion of newly developed green technologies. A discussion of appropriate strategies lies outside the scope of this theoretical work but should deserve a separate more empirically oriented treatment.

\section{References}

Barrett, S. (2003), Environment and Statecraft: The Strategy of Environmental TreatyMaking, Oxford University Press, Oxford UK et al.

Bergstrom, T., Blume, L. and Varian, H. (1986), On the private provision of public goods, Journal of Public Economics 29, 25-49.

Buchholz, W. and Konrad, K.A. (1994), Global environmental problems and the strategic choice of technology, Journal of Economics 60, 299-321.

Buchholz, W. and Peters, W. (2001), The overprovision anomaly of private public good supply, Journal of Economics 74, 63-78.

Buchholz, W. and Sandler, T. (2015), Successful leadership: Incorporating behavioural approaches into the theory of public good provision, mimeo.

Buchholz, W., Haslbeck, Ch. and Sandler, T. (1998), When does partial cooperation pay?, FinanzArchiv, 55, 1-20.

Cornes, R. and Hartley, R. (2007), Aggregative public good games, Journal of Public economic Theory 9, 201-219.

Cornes, R. and Sandler, T. (1996), The Theory of Externalities, Public Goods, and Club Goods, 2. Aufl., Cambridge University Press, Cambridge UK et al.

Cornes, R. and Sandler, T. (2000), Pareto-improving redistribution and pure public goods, German Economic Review 1, 269-286.

Finus, M. (2001), Game Theory and International Environmental Cooperation, Edward Elgar, Cheltenham UK and Northampton MA.

Finus, M. and Rübbelke, D. (2013), Public good provision and ancillary benefits: The case of climate agreements, Environmental and Resource Economics, 56, 211-226.

Hoel, M. (1991), Global environmental problems: The effects of unilateral action take by one country, Journal of Environmental Economics and Management 20, 55-70. 
Ihori, T. (1996), International public goods and contribution productivity differentials, Journal of Public Economics 61, 139-154.

Sandler, T. (2004), Global Collective Action, Cambridge University Press, Cambridge UK et al.

Sinn, H.-W. (2012), The Green Paradox - A Supply-Side Approach toGlobal Warming, The MIT Press, Cambridge MA and London UK.

Stern, N. (2015), Why are we Waiting? The Logic, Urgency, and Promise of Tackling Climate Change, The MIT Press, Cambridge MA and London UK.

Warr, P.G. (1982), The private provision of a public good is independent of the distribution of income, Economics Letters 13, 207-211. 